\title{
C'è un modo per evitare il sottoutilizzo della dialisi peritoneale, almeno in Italia?
}

\author{
Stefano Santarelli
}

U.O. di Nefrologia e Dialisi, Ospedale "Carlo Urbani”, Jesi (AN)

\begin{abstract}
IS THERE A WAY TO AVOID, AT LEAST IN ITALY, THE UNDERUTILISATION OF PERITONEAL DIALYSIS?
Abstract. Despite the cost of peritoneal dialysis (PD) is almost anywhere in the world lower than that of hemodialysis (HD), and survival studies reveal similar results between the 2 methods, DP is a much less used technique.

In the author's opinion one of the most important causes of this underutilisation in Italy is the poor knowledge of DP among nephrologists. It is thus suggested to give greater attention to the teaching of both the theory and practice of the method at Nephrology Specialization Schools and, where possible, to implement a turnover of nephrologists who manage patients in dedicated centers.
\end{abstract}

Key words: Peritoneal dialysis underutilisation, Nephrology Specialization School

Conflict of interest: None.

Financial support: None.

Accettato: 19 Agosto 2013

Nel 1999, Amedeo De Vecchi et al. (1) pubblicavano un'interessante "review" nella quale prendevano in esame, in 13 sistemi sanitari, i costi/rimborsi delle varie terapie sostitutive della funzione renale, escluso il trapianto. Con l'eccezione di Giappone e USA, essi erano molto simili: l'emodialisi (HD) nei centri pubblici risultava essere la metodica più costosa, seguita dalla stessa tecnica effettuata presso i centri privati e presso i centri ad assistenza limitata. Le meno costose erano l'HD domiciliare e la "continuous ambulatory peritoneal dialysis" (CAPD), mentre i costi della "automated peritoneal dialysis" (APD) erano simili a quelli di un'HD effettuata presso un centro ad assistenza limitata. In alcuni Paesi, due pazienti venivano trattati con la CAPD allo stesso costo di uno trattato con l'HD.

Nella discussione, gli Autori esprimevano l'opinione che i costi e/o i rimborsi influenzavano solo in parte la scelta della modalità terapeutica, mentre molto più importante era il rapporto costo-profitto per la struttura che offriva la terapia, e questo soprattutto per i centri privati che, per ammortizzare gli alti investimenti (infrastrutture, edifici e personale), avevano necessità di usare il centro in modo massimale.

Più recentemente, Baboolal et al. (2) hanno condotto uno studio multicentrico in 7 ospedali del Regno Unito per stimare i costi delle varie metodiche dialitiche, spinti anche dal fatto che, nel 2009, nel Paese era stato introdotto un nuovo sistema di finanziamento, il "Payment by Results" (PbR), con il quale diventava importante che le stime dei costi fossero corrette. L'analisi dei risultati mostrava che i costi, sia della APD che della CAPD, si rivelavano inferiori a quello dell'HD presso il centro ospedaliero rispettivamente del 28 $45 \%$ e del 47-61\%.

Gli Autori ritenevano sorprendente che, a dispetto di questi vantaggi, la percentuale dei pazienti trattati con la dialisi peritoneale (DP) nel Regno Unito era crollata dal 50\% dei primi anni '90 al 22\% del 2005 ed essi stessi prendevano in esame alcune possibili cause, senza arrivare a una sicura conclusione sulle reali responsabilità.

Nel 2006, Vonesh et al. (3) prendevano in esame 9 studi epidemiologici di sopravvivenza, tra i più recenti fino a quel momento, di pazienti trattati con la DP versus l'HD di vari Paesi e concludevano che la DP era associata dovunque ad una uguale o migliore sopravvivenza tra i pazienti non diabetici e tra quelli giovani. Per i pazienti più anziani, si evidenziavano delle differenze: negli USA l'HD era associata a una migliore sopravvivenza nei diabetici con età superiore ai 45 anni. In tutti gli studi, la DP mostrava una sopravvivenza equivalente o superiore nei primi uno-due anni di trattamento.

Per capire il perché, nonostante questi dati, la DP sia stata e sia così poco utilizzata, sono stati condotti vari studi in diversi Paesi (USA, Canada, Regno Unito). Il più recente è francese, pubblicato nel 2009 da Bouvier et al. (4), nel quale si chiedeva a nefrologi di cinque regioni della Francia di rispondere a un questionario. Le regioni erano state suddivise in 2 gruppi: 2 con una prevalenza di utilizzo della DP elevata $(>15 \%)$ e 3 con una prevalenza più bassa $(<10 \%)$. 
Il dato significativo che si è avuto da questo studio è quello di una diversa opinione, tra i due gruppi di nefrologi, sulla percentuale ottimale di pazienti prevalenti e incidenti in $\mathrm{DP}$, con valori più elevati tra coloro che lavoravano nelle aree geografiche con maggiore utilizzo e tra coloro che lavoravano nei centri pubblici rispetto ai privati.

Anche in Italia, nell'ultimo ventennio, ci siamo interrogati tantissimo, soprattutto nei convegni del Gruppo di Studio di Dialisi Peritoneale (GdSDP) della Società Italiana di Nefrologia (SIN), sul perché la DP sia così poco utilizzata nel nostro Paese. Le cause sono molteplici, ma io voglio porre l'attenzione solo su una di esse ed esporre in poche righe l'esperienza della mia Unità Operativa (UO).

A mio parere, la cultura "peritonealista" del nefrologo è determinante nella scelta di avviare o meno un paziente al trattamento dialitico peritoneale. La DP la si può scegliere solo se la si conosce bene e sono spesso la limitata esperienza o, addirittura, la non conoscenza che ne determinano l'esclusione dalle possibilità terapeutiche da offrire ai pazienti.

La non conoscenza parte da lontano: le Scuole di Specializzazione. Credo che, in Italia, siano veramente poche quelle che prevedano, nei loro piani di studio, di fornire un bagaglio culturale teorico-pratico adeguato. A mio parere quest'ultimo lo si può acquisire solo con una frequenza "sul campo" di almeno un anno, ma la maggior parte delle Scuole non la prevede ed è già molto se, nei 5 anni di corso, alcune settimane di lezioni e qualche esercitazione sono dedicate alla DP.

Un suggerimento che mi permetto di porgere al Consiglio Direttivo della SIN è di condurre un'indagine, magari coordinata dal GdSDP, sulle Scuole di Specializzazione in Nefrologia d'Italia, per verificare se e quanto è lontano dalla realtà ciò che sto sostenendo.

Per fare la DP bisogna crederci e, per crederci, la si deve conoscere; ne è la prova il fatto che, dove diventano Direttori di UO dei "peritonealisti", se questa metodica prima non si applicava, la si inizia ad applicare e, se la si adottava con una bassa prevalenza di pazienti, questa poi aumenta.

Una prova che per fare la DP bisogna crederci e che, per crederci, la si deve conoscere è data, a mio giudizio, dal fatto che, dove diventano Direttori di UO dei "peritonealisti", se questa metodica non la si applicava, la si inizia ad applicare e, se la si adottava con una bassa prevalenza di pazienti, essa aumenta.

Alcuni esempi.

Nella UO di Nefrologia di Trento, nel Marzo 2008, arrivava come Direttore un "peritonealista"; in quel momento la prevalenza della DP era del 10\%; attualmente è del $18 \%$, con un'incidenza, nei primi mesi del 2013, del 48\% (dati cortesemente forniti dal Dr. Giuliano Brunori).

Nel Dicembre del 2008 arrivava a Bassano del Grappa (VI) un altro Direttore "peritonealista" e, da allora a oggi (Agosto 2013), la prevalenza dei pazienti in peritoneale è passata dal $3.4 \%$ al $26.6 \%$ (dati cortesemente forniti dal Dr. Roberto Dell'Aquila che, con l'invio del dato, mi ha scritto: "Mi è costata una fatica pazzesca cambiare la mentalità dei miei Collaboratori, dell'Amministrazione, dei Medici di base e dei Pazienti ma non ho cambiato la mia!!!").

Nel Gennaio del 2013 diventava Direttore, a San Daniele del
Friuli (UD), un altro "peritonealista" e, in 8 mesi, l'incidenza della DP passava dallo $0 \%$ al $57 \%$ (dati cortesemente forniti dal Dr. Gianpaolo Amici).

Un altro fatto, a mio giudizio, merita una riflessione: nella quasi totalità delle UO che effettuano la DP, essa è seguita solo da un medico (eccezionalmente due, nei centri più grandi). Se questo medico dovesse trasferirsi ad un altro centro è molto probabile che, nel primo, incidenza e prevalenza dei pazienti in DP crollerebbero.

Nella UO da me diretta, dal 1995 fino al Marzo del 2006, i pazienti in DP venivano seguiti da me con l'aiuto di uno specializzando. Nel Marzo del 2006, dovendo entrambi abbandonare la gestione diretta di quei pazienti, presi la decisione, in accordo con tre miei Collaboratori, di effettuare una loro rotazione ogni sei mesi, naturalmente sotto tutoraggio. Dopo due anni, tutti e tre i Collaboratori erano diventati completamente autonomi, la prevalenza è passata dal 31\% del 2006 al $35 \%$ di oggi e la DP è considerata la metodica di prima scelta per ogni paziente.

In conclusione, naturalmente non intendo sostenere che tutti i Direttori di UO di Nefrologia debbano essere "peritonealisti" (al riguardo, penso che colui che viene nominato Direttore di UO debba essere esclusivamente il più competente e meritevole tra i candidati), ma sono convinto che la conoscenza sia l'arma vincente per un maggiore e migliore utilizzo della DP. E la conoscenza della DP, con la conseguente fiducia in essa, dovrebbe essere di tutta l'équipe medica e di quasi tutta l'équipe infermieristica.

\section{Riassunto}

Nonostante i costi della dialisi peritoneale (DP) siano più bassi di quelli dell'emodialisi (HD), un po' dovunque nel mondo, e gli studi di sopravvivenza evidenzino risultati sovrapponibili tra le due metodiche, la DP è una tecnica molto meno utilizzata.

Una delle cause più importanti di tale modesto impiego è, almeno in Italia, la scarsa conoscenza della DP tra i nefrologi.

Vengono suggerite una maggiore attenzione all'insegnamento teorico-pratico della metodica nelle Scuole di Specializzazione in Nefrologia e una rotazione, dove possibile, dei medici nefrologi che gestiscono i pazienti trattati con la DP nei centri.

Parole chiave: Sottoutilizzo della dialisi peritoneale, Scuola di specializzazione nefrologica

Dichiarazione di conflitto di interessi: L'Autore dichiara di non avere conflitto di interessi.

Contributi economici degli autori: L'Autore dichiara di non aver ricevuto sponsorizzazioni economiche per la preparazione dell'articolo. 
Indirizzo dell'Autore:

Dr. Stefano Santarelli

U.O. Nefrologia e Dialisi

Ospedale "Carlo Urbani"

Via dei Colli, 52

60035 Jesi (AN)

stefano.santarelli@sanita.marche.it

\section{Bibliografia}

1. De Vecchi AF, Dratwa M, Wiedemann ME. Healthcare systems and end-stage renal disease (ESRD) therapies--an international review: costs and reimbursement/funding of ESRD therapies. Nephrol Dial Transplant 1999; 14 (Suppl. 6): 31-41.

2. Baboolal K, McEwan P, Sondhi S, Spiewanowski P, Wechowski J, Wilson $\mathrm{K}$. The cost of renal dialysis in UK setting--a multicentre study. Nephrol Dial Transplant 2008; 23: 1982-9.

3. Vonesh EF, Snyder JJ, Foley RN, Collins AJ. Mortality studies comparing peritoneal dialysis and hemodialysis: What do they tell us? Kidney Int 2006; 70: S3-11.

4. Bouvier N, Durand PY, Testa A, et al. Regional discrepancies in peritoneal dialysis utilization in France: the role of the nephrologist's opinion about peritoneal dialysis. Nephrol Dial Transplant 2009; 24: 1293-7. 